\title{
HPV VACCINATION AWARENESS AND ITS RELATION TO ORAL AND OROPHARYNGEAL CANCER: A SINGLE CENTRE STUDY
}

1. Sreejee Gopalakrishnan,

Resident, Department of Oral and Maxillofacial Surgery, Faculty of Dental Sciences, Sri Ramachandra Institute of Higher Education and Research

MOBILE: 9444757370

E-MAIL ID: sreejeegopalakrishnan@gmail.com

\section{S. Elengkumaran,}

Associate Professor, Department of Oral and Maxillofacial Surgery, Faculty of Dental Sciences, Sri Ramachandra Institute of Higher Education and Research

MOBILE: 9944943451

E-MAIL ID: drkumaranomfs@ gmail.com

3. S. Poojyashree, Under-graduate,

Faculty of Dental Sciences, Sri Ramachandra Institute of Higher Education and Research

MOBILE: 8939616515

E-MAIL ID: poojyasundar174@gmail.com

\section{Pooja .K.N,}

Under-graduate,

Faculty of Dental Sciences, Sri Ramachandra Institute of Higher Education and Research

MOBILE: 7338969774

E-MAIL ID: k.n.pooj@gmail.com

\begin{abstract}
Background - The recently developed HPV vaccine is highly effective against the HPV virus. It has met widespread acceptance amongst healthcare and public health professionals. However, there are still social barriers to vaccination that hampers the effects of preventing the disease caused by HPV. The primary goal of this study was to evaluate the survey responses regarding the knowledge, awareness, and vaccination status among dental professionals and students in a local population and to emphasize the relationship between HPV and the increasing incidence of oral and oropharyngeal cancers in the current scenario.
\end{abstract}

Methodology - This study was a prospective analysis of a questionnaire collected from the dentist and dental students at The Faculty of Dental Sciences, Sri Ramachandra Institute of Higher Education and Research, Porur, Chennai, Tamilnadu, India; regarding their knowledge, awareness, and Human Papilloma Virus (HPV) - vaccination status. 
Results - 96\% of the participants were aware of the HPV and only 4\% were unaware of the virus. About $18.7 \%$ of the participants were unaware of the modes of transmission of HPV. Only $66.6 \%$ knew that HPV - 16 and 18 were responsible for Oral and cervical cancers.

Conclusion - Understanding the barriers and hesitancy to vaccination is the crucial step to designing strategies that may aid in enhancing the vaccination

Keywords - Human Papilloma Virus, HPV vaccine, Oral and oropharyngeal cancer

\section{INTRODUCTION}

HPV is a non-enveloped, double-stranded, circular DNA virus associated with various genital and aero-digestive diseases, including warts, laryngeal papilloma, and cervical cancer ${ }^{4}$. Although most HPV infections resolve spontaneously, relentless infection by Human Papilloma Virus (HPV) strains 16 and 18 can cause Oral and cervical cancers ${ }^{1}$. HPV 16 and 18 are globally responsible for $85 \%$ of head and neck carcinomas ${ }^{5}$. Three cancer locations in the head and neck have been linked with HPV: oropharynx, oral cavity, and larynx ${ }^{5}$. Around 30\% of oropharyngeal cancers are caused by $\mathrm{HPV}^{5}$. The prevalence of HPV infection and oral cancer is significantly higher throughout the world ${ }^{1}$. The preponderance of these HPV strains can vary by way of socio-economic factors, including the geography and the ethnic group affected $^{1}$.

Recent systematic reviews and meta-analyses have shown convincing evidence of significant reductions in HPV infections in geographical areas with increased vaccination, which will possibly facilitate both clinical protection and herd immunity within that population ${ }^{6}$.

The World Health Organization (WHO) has necessitated the global elimination of oral and cervical cancer as it is preventable with a well-known etiological factor. The various levels of prevention available to prevent this type of cancer are - primary prevention by HPV vaccination and sexual education, secondary prevention by screening and treatment of carcinoma in situ, and tertiary prevention using timely diagnosis and prompt treatment. Primary prevention with vaccines is pertinent to young adolescent girls, and secondary prevention with screening is for women ${ }^{7}$.

Though the HPV vaccination is a successful public health intervention, the level of community protection achieved through it is still considerably lower ${ }^{1}$. HPV vaccination rates are still lower regardless of the availability of vaccines with well-known safety and efficacy ${ }^{1}$. Measuring the rates of vaccination can be challenging and entail different measures to quantify the impact $(\text { Table }-1)^{1}$.

The common barriers to vaccine acceptability are socio-demographic characteristics (e.g., age, education, race, marital status, pregnancy (or trying to conceive)), awareness and dogmas (e.g., perceived lack of need, religious or community influences, fear of side effects), competing priorities (e.g., child-care, funding), apprehension about the vaccination process (e.g., pain or fear of needle pricks) ${ }^{1}$. Other challenges are information and consent process, vaccination 
setting, anxiety regarding privacy and pain during vaccination, financial constraints, social norms, and values ${ }^{1}$.

Only a small number of studies have evaluated the familiarity, understanding, and acceptance of HPV vaccination among undergraduate dental students, post-graduate residents, and practitioners. These studies have evaluated and found deficiencies in both knowledge and awareness among dental students specifically, that may be added to the dental curriculum ${ }^{6}$. This study was a prospective analysis of a questionnaire collected from the dentist and dental students at The Faculty of Dental Sciences, Sri Ramachandra Institute of Higher Education and Research, Porur, Chennai, Tamilnadu, India; regarding their knowledge, awareness and HPV vaccination status.

\section{MATERIALS AND METHODS}

This study was a prospective analysis of a questionnaire collected from the dentist and dental students at The Faculty of Dental Sciences, Sri Ramachandra Institute of Higher Education and Research, Porur, Chennai, Tamilnadu, India; regarding their knowledge, awareness, and Human Papilloma Virus (HPV) - vaccination status. A total of 10 questions were framed and circulated among the dental students and faculties as Multiple-Choice Questions (MCQs) through a Google form for them to fill in (Figure 1).

\section{RESULTS}

The questionnaire was circulated in a randomized manner to 250 members. $96 \%$ of the participants were aware of the HPV and only $4 \%$ were unaware of the virus. $76.4 \%$ of the participants were aware of the modes of transmission of HPV and $18.7 \%$ were not sure about its mode of transmission and $4.9 \%$ of the participants did not know the route of transmission of HPV Only $66.3 \%$ of the participants knew that HPV-16 causes oral and cervical cancer. $76.4 \%$ of the participants were aware that health care professionals are at risk of getting affected by HPV. $72.8 \%$ of the participants were aware of HPV vaccination. Only $82 \%$ of the participants were vaccinated for HPV. $82 \%$ considered the vaccination to be safe and necessary.

According to the data collected $15.2 \%$ of participants felt that there is lack of knowledge about the vaccine, $6 \%$ felt it's due to religious beliefs, $39.2 \%$ feared complications/side effects of the vaccine and $39.6 \%$ felt there may be any other causes like age range for the administration of the vaccine. Only $70.8 \%$ of the participants were aware of the vaccination guidelines for HPV (Figure 2). 96\% of the participants felt that there is a need for awareness about HPV and vaccination against HPV (Figure 3 ).

\section{DISCUSSION}

Oral carcinoma, with an incidence rate of 16.1 adults per 1 lakh, is one of the leading malignancies worldwide with a varied geographic distribution ${ }^{3}$. Squamous cell carcinoma (SCC) is the most common oral malignancy ${ }^{3}$. The percentage of carcinomas of the tongue and palatine tonsils is on the rise among younger men $(2-4 \%)^{2}$. This increased prevalence of a 
subdivision of oropharyngeal carcinoma can be associated with Human Papilloma Virus (HPV) ${ }^{2}$. Among the head and neck cancers, a strong association with HPV infection is evident with oropharyngeal cancers, particularly tonsillar and basal tongue cancers ${ }^{2}$. HPV-related carcinomas accounted for about $4.5 \%$ of all cancers ${ }^{5}$. These represent $8.6 \%$ of cancers in women and $0.8 \%$ in men and account for $6.7 \%$ of all cancers in low- and middle-income countries and $2.8 \%$ in high-income countries ${ }^{5}$. In 2012, of 636,000 HPV-related cancers worldwide, approximately 38,000 were head and neck cancers ${ }^{5}$.

Two vaccines approved globally are available in India; a quadrivalent vaccine (GardasilTM marketed by Merck) and a bivalent vaccine (Cervarix TM marketed by Glaxo Smith Kline). Both of these vaccines are made by recombinant DNA technology that produces non-infectious virus-like particles (VLPs) comprising of the HPV L1 protein ${ }^{9}$. An Indian quadrivalent HPV vaccine, which includes VLPs of HPV types 6,11, 16, and 18 made by Serum Institute of India (Pune, India) is currently going through phase 2-3 trials for effectiveness and safety ${ }^{8}$.

$70 \%-90 \%$ of all HPV-related carcinomas can be prevented by universal high-coverage HPV vaccination ${ }^{5}$. Universal vaccination of girls is the most effective measure to evade cases of and deaths from carcinomas caused by $\mathrm{HPV}^{5}$.

\section{CONCLUSION}

Understanding the barriers and hesitancy to vaccination is the crucial step to designing strategies that may aid in enhancing the vaccination. Three effective methods - vaccination against HPV, screening for cancer followed by treatment of Carcinoma in situ will be highly beneficial in eliminating oropharyngeal cancer in India and worldwide.

\section{CONFLICT OF INTEREST}

None

\section{REFERENCES}

1. Mrklas KJ, MacDonald S, Shea-Budgell MA, Bedingfield N, Ganshorn H, Glaze S, Bill L, Healy B, Healy C, Guichon J, Colquhoun A. Barriers, supports, and effective interventions for uptake of human papillomavirus-and other vaccines within global and Canadian Indigenous peoples: a systematic review protocol. Systematic reviews. 2018 Dec;7(1):1-20.

2. Woo SB, Cashman EC, Lerman MA. Human papillomavirus-associated oral intraepithelial neoplasia. Modern Pathology. 2013 Oct;26(10):1288-1297.

3. Prakash P, Khandare M, Kumar M, Khanna R, Singh GP, Nath G, Gulati AK. Immunohistochemical detection of p16INK4a in leukoplakia and oral squamous cell carcinoma. Journal of Clinical and Diagnostic Research: JCDR. 2013 Dec;7(12):2793-5.

4. Ha PK, Califano JA. The role of human papillomavirus in oral carcinogenesis. Critical Reviews in Oral Biology \& Medicine. 2004 Jul;15(4):188-96.

5. de Martel C, Plummer M, Vignat J, Franceschi S. Worldwide burden of cancer attributable to HPV by site, country and HPV type. International Journal of Cancer 2017;141(4):664-70. 
6. Mann SK, Kingsley K. Human Papillomavirus (HPV) Vaccine Knowledge, Awareness and Acceptance among Dental Students and Post-Graduate Dental Residents. Dentistry journal. 2020 Jun;8(2):45.

7. Pampapati V, Anantharaju A. Human papillomavirus vaccination: An important tool in cervical cancer elimination. International Journal of Advanced Medical and Health Research. 2020 Jan $1 ; 7(1): 3$.

8. Sankaranarayanan R, Basu P, Kaur P, Bhaskar R, Singh GB, Denzongpa P, Grover RK, Sebastian P, Saikia T, Oswal K, Kanodia R. Current status of human papillomavirus vaccination in India's cervical cancer prevention efforts. The Lancet Oncology. 2019 Nov 1;20(11):e637-44.

9. Kaarthigeyan K. Cervical cancer in India and HPV vaccination. Indian journal of medical and paediatric oncology: official journal of Indian Society of Medical \& Paediatric Oncology. 2012 Jan;33(1):7.

\section{FIGURES}

\section{Figure 1 - Questionnaire}

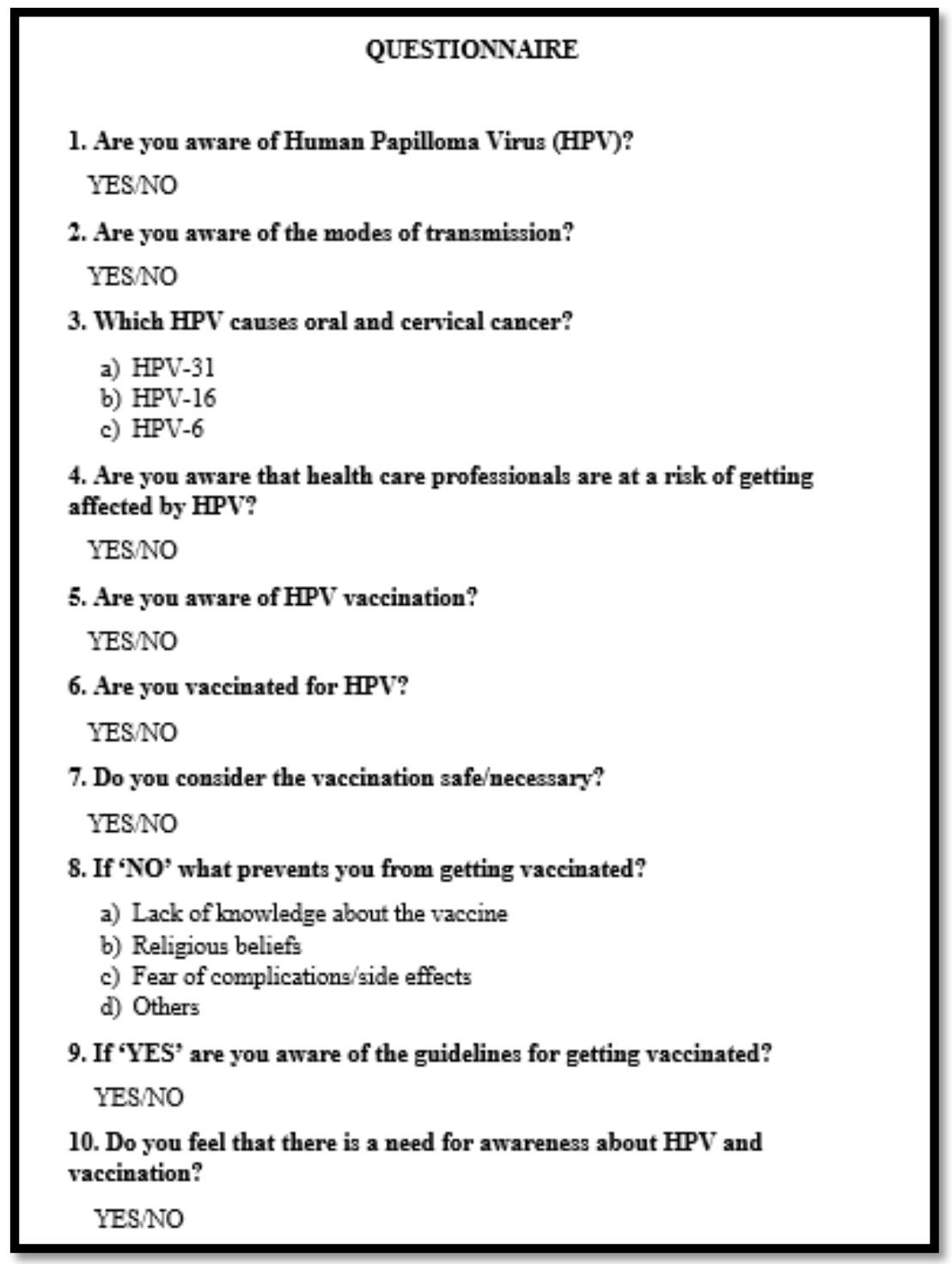


Figure 2 - Responses for "Are you vaccinated for HPV?"

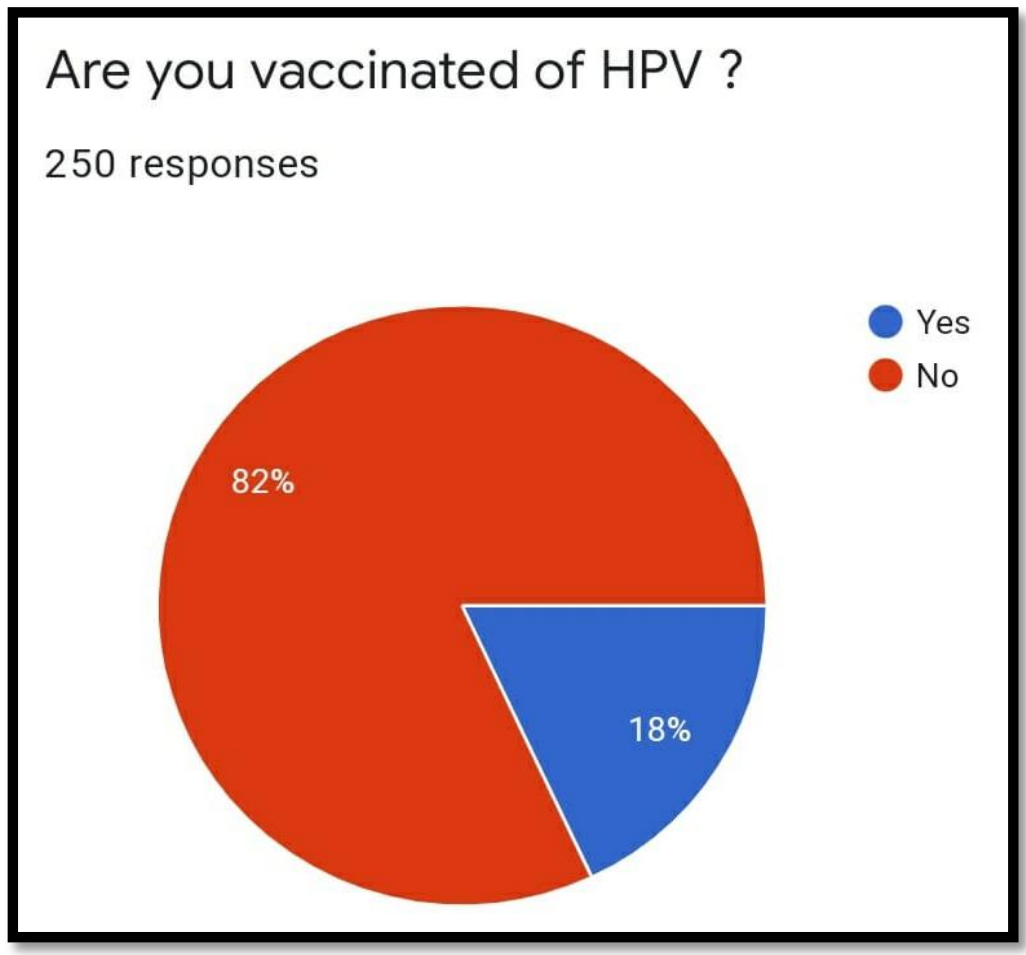

Figure 3 - Responses for "Do you feel there is a need for awareness about HPV vaccination?"

Do you feel that there is a need for awareness about HPV and vaccination?

250 responses 